\title{
Eyelid Sebaceous Gland Carcinoma
}

National Cancer Institute

\section{Source}

National Cancer Institute. Eyelid Sebaceous Gland Carcinoma. NCI Thesaurus. Code C134831.

A sebaceous gland carcinoma affecting the eyelid. It arises from the meibomian glands, glands of Zeis, or glands associated with the caruncle. It usually affects elderly women and is characterized by high rate of local recurrence, regional, and distant metastases. 